This item was submitted to Loughborough's Research Repository by the author.

Items in Figshare are protected by copyright, with all rights reserved, unless otherwise indicated.

\title{
Quantum Monte Carlo computation: The sign problem as a Berry phase
}

PLEASE CITE THE PUBLISHED VERSION

PUBLISHER

(C) American Physical Society

LICENCE

CC BY-NC-ND 4.0

REPOSITORY RECORD

Samson, J.H.. 2019. "Quantum Monte Carlo Computation: The Sign Problem as a Berry Phase”. figshare. https://hdl.handle.net/2134/1354. 


\title{
Quantum Monte Carlo computation: The sign problem as a Berry phase
}

\author{
J. H. Samson \\ Department of Physics, University of Technology, Loughborough, Leicestershire, LE11 3TU, England
}

(Received 21 July 1992)

\begin{abstract}
The partition function of interacting electrons is often represented as that of noninteracting electrons moving in a stochastic time-dependent field, integrated over the field. Quantum Monte Carlo computation of this functional integral suffers from the sign problem: rapid oscillation of the sign of the integrand at low temperature. The integrand is in general complex: the phase tends to a Berry phase for smooth paths at low temperatures, where spins follow fields adiabatically, and to zero in the high-temperature limit, recovering the static approximation.
\end{abstract}

The collective behavior of correlated electrons has been studied extensively for many years. The center of interest has shifted from magnetism via heavy fermions to high-temperature superconductivty. A widely used method to study such behavior eliminates electronic degrees of freedom, leaving an effective Hamiltonian for an auxiliary field. ${ }^{1}$ The system is described by a functional integral over this imaginary-time-dependent field, usually related to an order parameter. In recent years it has become possible to compute such integrals by quantum Monte Carlo methods. An unfortunate complication plaguing quantum Monte Carlo calculations in fermion systems is the sign problem: the integrand is frequently not positive definite at low temperatures. Averages become differences of two positive quantities of similar magnitude, and the numerical problem becomes unstable. In the systems described here, the integrand is not necessarily real and the sign problem becomes a phase problem.

This paper proposes a geometrical interpretation of this problem. ${ }^{2}$ In the functional integral, an auxiliary field transports the electron system along a path in state space. A wave function, transported adiabatically around a closed path, develops a Berry phase ${ }^{3}$ in addition to the naively expected dynamical phase. The adiabatic condition holds for smooth paths at low temperatures. The integrand therefore has a phase factor, and it is this phase that is responsible for the sign problem in systems such as the Heisenberg model and $N$-band Hubbard model. At high temperatures there is insufficient imaginary time for the fermions to follow the field adiabatically; they respond only to the time-averaged field. Adiabatic transport is also the basis of the Born-Oppenheimer approximation. Many systems separate to a greater or lesser extent into "fast" and "slow" variables. In a molecule, the electronic ground state is determined for fixed nuclear configurations, and its energy appears in an effective nuclear potential. Near an electronic degeneracy this is insufficient: a vector potential must be added to the nuclear Hamiltonian. ${ }^{4}$ In the Hubbard model it is also often possible to separate "fast" electron hopping from "slow" spin fluctuations. Similar corrections have already been pointed out for spin fluctuations. 5

The Hamiltonian for electrons with two-body interac- tions (in a single-particle basis $\{|\alpha\rangle\}$ ) is

$$
H=H_{\text {band }}+H_{I} \text {, }
$$

where

$$
H_{\text {band }}=\sum_{\alpha, \beta} t_{\alpha \beta} c_{\alpha}^{\dagger} c_{\beta}
$$

and

$$
H_{I}=\frac{1}{2} \sum_{\alpha, \beta, \gamma, \delta} v_{\alpha \beta, \gamma \delta} c_{\alpha}^{\dagger} c_{\beta} c_{\gamma}^{\dagger} c_{\delta} .
$$

The coefficients are taken as real. The HubbardStratonovich transformation 1,7 replaces the electronelectron interaction by an interaction between electrons and an imaginary-time-dependent auxiliary field with matrix elements $u_{\alpha \beta}(\tau)$. This is typically an exchange field, acting as a Zeeman splitting on the spins. The "partition function" of electrons in this field, $z[u]$, is averaged over Gaussian fluctuations of the field in a functional integral

$$
\begin{aligned}
Z & =\frac{1}{\mathcal{N}} \int \prod_{\alpha \beta} \mathcal{D} u_{\alpha \beta}(\tau) e^{-\beta V[u]} \\
& =\frac{1}{\mathcal{N}} \int \prod_{\alpha \beta} D u_{\alpha \beta}(\tau) e^{-\beta V_{0}[u]} z[u],
\end{aligned}
$$

where

$V_{0}[u]=-k T \int_{0}^{\beta} \sum_{\alpha, \beta, \gamma, \delta} \frac{1}{2} u_{\alpha \beta}(\tau) v_{\alpha \beta, \gamma \delta}^{-1} u_{\gamma \delta}(\tau) d \tau$,

and

$$
z[u]=\operatorname{Tr} U[u ; \beta],
$$

where

$$
\begin{gathered}
U\left[u ; \tau_{1}\right]=\mathcal{T} \exp \int_{0}^{\tau_{1}} \sum_{\alpha, \beta}\left[-t_{\alpha \beta}-u_{\alpha \beta}(\tau)+\mu \delta_{\alpha \beta}\right] \\
\times c_{\alpha \tau}^{\dagger} c_{\beta \tau} d \tau
\end{gathered}
$$

$z[u]$ will be called the weight of the path $u(\tau), V[u]$ the effective potential, and $U$ the (nonunitary) evolution operator. $\mathcal{T}$ is the imaginary-time-ordering symbol and $\mathcal{N}$ the normalization. In cases studied here, $v$ is negative definite so that the Gaussian in Eq. (4) is bounded. 
The static approximation restricts integration to timeindependent fields:

$$
\begin{aligned}
Z_{\mathrm{st}} & =\frac{1}{\mathcal{N}^{\prime}} \int \prod_{\alpha \beta} d u_{\alpha \beta} e^{-\beta V_{\mathrm{st}}(u)} \\
& =\frac{1}{\mathcal{N}^{\prime}} \int \prod_{\alpha \beta} d u_{\alpha \beta} \exp \left[\beta \sum_{\alpha, \beta, \gamma, \delta} \frac{1}{2} u_{\alpha \beta} v_{\alpha \beta, \gamma \delta}^{-1} u_{\gamma \delta}\right] z_{\mathrm{st}}(u)
\end{aligned}
$$

where

$$
z_{\mathrm{st}}(u)=\operatorname{Tr} \exp \left(-\beta \sum_{\alpha, \beta}\left(t_{\alpha \beta}+u_{\alpha \beta}-\mu \delta_{\alpha \beta}\right) c_{\alpha}^{\dagger} c_{\beta}\right]
$$

is the partition function of electrons in a static field. Many authors use electronic structure techniques to evaluate $V_{\text {st }}$ for a range of configurations and carry out statistical mechanics on this effective Hamiltonian. Recent such work found a spin correlation function in iron almost identical to that in the classical nearest-neighbor Heisenberg model, ${ }^{8}$ and it was suggested that quantum corrections might modify this result. This was a motivation for the present work.

The functional integral (4) and expectation values have been studied by quantum Monte Carlo techniques. ${ }^{9,10}$ Classical Monte Carlo simulation samples configurations $p$ from a distribution $w_{p}$, such as the integrand in Eq. (8), and computes averages of the form $\langle A\rangle$ $=\Sigma_{p}\left(w_{p} A_{p}\right) / \Sigma_{p}\left(w_{p}\right)$. In a quantum simulation the integrand in Eq. (4) provides the distribution. Unfortunately, this is not positive definite; the average sign can vanish as $T \rightarrow 0 .^{9}$ Averages calculated in this way are formally correct but numerically extremely unstable. This is the sign problem.

The interest is in the sign or phase of the weight $z[u]$, since the Gaussian factor $\exp \left(-\beta V_{0}\right)$ is positive. For certain Hamiltonians (such as a one-band Hubbard model) $z$ is real, but it is in general complex if the field breaks time-reversal invariance. For each field $u$ there is a conjugate field $\tilde{u}_{\alpha \beta}(\tau)=\left[u_{\beta \alpha}(\beta-\tau)\right]^{*}$. The weight of $\tilde{u}$ is the complex conjugate of the weight of $u: z[\tilde{u}]=z[u]^{*}$. Since both a path and its conjugate appear in the integration with the same $V_{0}$, only the real part of $z[u]$ is needed; if $u$ is a real symmetric matrix, the weight its real.

In the one-band Hubbard model

$$
H=H_{\mathrm{band}}+I \sum_{i} n_{i \uparrow} n_{i \downarrow}=H_{\mathrm{band}}+\frac{1}{2} I n-2 I \sum_{i} S_{i z}^{2},
$$

the single-particle operators appearing in the interaction term commute. There is considerable freedom in splitting this term, but one can choose the field $u_{\alpha \beta}$ to be a real symmetric matrix coupling to $S_{i z}$ [with suppression of site indices, $\quad u_{\uparrow \uparrow}=-\Delta(\tau) / 2, \quad u_{\downarrow \downarrow}=\Delta(\tau) / 2$, $\left.u_{\uparrow \downarrow}=u_{\downarrow \uparrow}=0\right]$. The weight is real. The origin of the sign problem is well understood in this case: at low temperature there is sufficient time for the evolution to exchange electrons, each interchange giving a minus sign. ${ }^{9}$ The problem has recently been studied in terms of dynamics on the manifold of Slater determinants. The stochastic fields induce a diffusion equation; ${ }^{11}$ at long times a Slater determinant $|\psi\rangle$ can diffuse to the "opposite side" of the manifold, giving negative overlap $\langle\psi|U[u ; \beta]| \psi\rangle$. The sign of the overlap has been interpreted as a topological invariant: the rotation of the one-electron basis needed to restore the final Slater determinant to the initial state is either proper or improper. ${ }^{12}$

In general one cannot restrict attention to real fields. The one-band Hubbard model (10) is isotropic in spin space, but an $N$-band generalization (for transition metals) requires a manifestly isotropic interaction term such as $\sum_{i} I \mathbf{S}_{i} \cdot \mathbf{S}_{i}$. The auxiliary field is then a vector field

$$
\sum_{\alpha, \beta} u_{\alpha \beta}(\tau) c_{\alpha}^{\dagger}(\tau) c_{\beta}(\tau)=-\sum_{i} \Delta_{i}(\tau) \cdot \mathbf{S}_{i}(\tau)
$$

The Hamiltonian is no longer real, and the components of the field no longer commute. The use of vector fields has been criticized on the grounds that an expansion contains diagrams violating the Pauli principle. ${ }^{13}$ However, in an $N$-band Hubbard model it seems that a cure would either break isotropy or require $N$ exchange fields per atom.

A discussion of geometrical phases involves dynamics in three spaces: the auxiliary field traces out a field path in parameter space, the wave function evolves in Hilbert space $\mathcal{H}$, and the state traces out what shall be referred to as a spin path in state space $\mathcal{P}$. A point $\mathbf{n} \in \mathcal{P}$ corresponds to the ray $c|\mathbf{n}\rangle$ in Hilbert space, where $c$ is a nonzero complex number. All such wave functions correspond to the same physical state.

The result will be derived first for the spin- $\frac{1}{2}$ Heisenberg model

$$
H=-\sum_{i j} J_{i j} \mathbf{S}_{i} \cdot \mathbf{S}_{j} .
$$

The partition function is of the form ${ }^{14}$

$$
\begin{aligned}
Z=\frac{1}{\mathcal{N}} \int \prod_{i} & \mathcal{D}^{3} \Delta_{i}(\tau) \exp \left[-\int_{0}^{\beta} \sum_{i j} \frac{1}{4} \Delta_{i}(\tau) \cdot J_{i j}^{-1} \Delta_{j}(\tau) d \tau\right) \\
& \times z[\Delta],
\end{aligned}
$$

with

$$
\begin{aligned}
& z[\Delta]=\operatorname{Tr} U[\Delta ; \beta], \\
& \quad \text { where } U\left[\Delta ; \tau_{1}\right]=\prod_{i} \mathcal{T} \exp \left[\int_{0}^{\tau_{1}} \Delta_{i}(\tau) \cdot \mathbf{S}_{i \tau} d \tau\right) .
\end{aligned}
$$

(Functional integration over coherent spin states ${ }^{15}$ gives similar results. Coherent-state integration is less practical in the Hubbard model because of the large number of degrees of freedom.) Since the weight $z[\Delta]$ is a product of single-site terms, only a single spin need be considered. In that case the parameter space is $\mathbb{R}^{3}$, the Hilbert space is the space of two-component spinors, and the state space $\mathcal{P}$ is the sphere $S^{2}$ : the unit vector $\mathbf{n}$ defines the spin direction. One chooses a normalized state $|\mathbf{n}\rangle \in \mathscr{H}$ for each $\mathbf{n}$, with $\mathbf{n} \cdot \boldsymbol{\sigma}|\mathbf{n}\rangle=|\mathbf{n}\rangle$, by making a choice of phase such as

$$
|\mathbf{n}\rangle=\left(\begin{array}{c}
\cos (\theta / 2) e^{-i \phi / 2} \\
\sin (\theta / 2) e^{i \phi / 2}
\end{array}\right) \text {. }
$$

The evolution operator $U[\Delta ; \tau]$ of a static field can be 
resolved into projection operators $P(\mathbf{n})=|\mathbf{n}\rangle\langle\mathbf{n}|$ :

$$
e^{\Delta \cdot \sigma \tau / 2}=e^{\Delta \tau / 2} P(\widehat{\Delta})+e^{-\Delta \tau / 2} P(-\widehat{\Delta}) .
$$

The spin $\mathbf{n}$ relaxes towards the $\Delta$ direction: $\tan (\alpha / 2) \propto e^{-\Delta \tau}$, with $\alpha$ the angle between $\Delta$ and $\mathbf{n}$. For a time-dependent field this becomes a pursuit problem on the sphere: $\mathbf{n}$ relaxes towards the instantaneous field direction. If $\beta \Delta$ exceeds a typical angular velocity of the field, the spin will follow nearly adiabatically. Now a spin describing a closed curve develops a phase shift. ${ }^{3}$ Aharnov and Anandan ${ }^{16}$ relate the phase to a (not necessarily adiabatic) cyclic evolution of the state in $\mathcal{P}$. The latter argument, adapted here to imaginary time, provides an expression for the amplitude and phase factors of an arbitrary field path. The evolution operator has two eigenstates, which describe closed spin paths:

$$
|\psi(\beta)\rangle=U[\Delta ; \beta]|\psi(0)\rangle=R e^{i \Phi}|\psi(0)\rangle
$$

Since $\operatorname{det} U=1$, the weight in Eq. (14) is $z[\Delta]=R e^{i \Phi}+R^{-1} e^{-i \Phi}$. The amplitude $R$ and phase $\Phi$ will now be determined. The wave function obeys the imaginary-time Schrödinger equation, with $H(\tau)=-\Delta(\tau) \cdot \sigma / 2$ :

$$
H(\tau)|\psi(\tau)\rangle=-\frac{\partial}{\partial \tau}|\psi(\tau)\rangle
$$

Now any nonzero spinor corresponds to a unique state $|\mathbf{n}\rangle$, so that

$|\psi(\tau)\rangle=c(\tau)|\mathbf{n}(\tau)\rangle$, with $c(\beta) / c(0)=R e^{i \Phi}$.

Substituting this in the Schrödinger equation (18) gives

$$
\begin{aligned}
& |c(\tau)|^{2}\langle\mathbf{n}(\tau)|H(\tau)| \mathbf{n}(\tau)\rangle=-\langle\mathbf{n}(\tau)| c^{*}(\tau) \frac{\partial}{\partial \tau}[c(\tau)|\mathbf{n}(\tau)\rangle] \\
& \Longrightarrow\langle\mathbf{n}(\tau)|H(\tau)| \mathbf{n}(\tau)\rangle=-\frac{1}{c} \frac{d c}{d \tau}-\left\langle\mathbf{n}(\tau)\left|\frac{\partial}{\partial \tau}\right| \mathbf{n}(\tau)\right\rangle \\
& =\int_{0}^{\beta}\langle\mathbf{n}(\tau)|H(\tau)| \mathbf{n}(\tau)\rangle d \tau \\
& =[-\ln c(\tau)]_{0}^{\beta}-\oint\langle\mathbf{n}(\tau)|\nabla| \mathbf{n}(\tau)\rangle \cdot d \mathbf{n} .
\end{aligned}
$$

This line integral is a geometrical property of the spin path; its value is $-i \Omega / 2$, where $\Omega$ is the solid angle enclosed by the spin path. ${ }^{3}$ Substituting the dynamical quantities gives

$$
\int_{0}^{\beta}-\frac{1}{2} \Delta(\tau) \cdot \mathbf{n}(\tau) d \tau=-\ln R-i \Phi+i \Omega / 2 .
$$

In contrast to unitary (real-time) quantum evolution, the dynamical and geometrical contributions separate unambiguously as real and imaginary parts of (21). The amplitude is

$$
\begin{aligned}
R & =\exp \left[\int_{0}^{\beta} \frac{1}{2} \Delta(\tau) \cdot \mathbf{n}(\tau) d \tau\right] \\
& \leq \exp \left[\int_{0}^{\beta} \frac{1}{2}|\Delta(\tau)| d \tau\right]
\end{aligned}
$$

and the phase is $\Phi=\Omega / 2$. This result is exact; no adiabatic assumption is made. The inequality (22) becomes an equality in the adiabatic case. The phase factor also appears as a Wess-Zumino term in the coherent-state functional integral, ${ }^{15}$ has been noted elsewhere in the auxiliary-field functional integral. ${ }^{5,6}$ The nonintegrability of the problem means that the weight has no simple functional form, although it can be solved exactly for a conical field path

$$
\Delta(\tau)=\Delta(\sin \theta \cos 2 \pi n \tau / \beta, \sin \theta \sin 2 \pi n \tau / \beta, \cos \theta),
$$

in which case the spin path is a narrower cone, coaxial with the field path, and the weight (for integer $n$ ) is

$$
z[\Delta]=2(-1)^{n} \cosh \left[\frac{1}{4}(\beta \Delta)^{2}-n^{2} \pi^{2}+i n \beta \Delta \pi \cos \theta\right]^{1 / 2} .
$$

The weight of an arbitrary path is complex. However, since paths appear in complex conjugate pairs, only the sign of the real part of the weight is important. At low temperatures, there is sufficient imaginary time $\beta$ for the spin to follow a smooth field path nearly adiabatically and enclose a solid angle $\gg \pi$, giving an arbitrary sign. At high temperatures, since the typical $\Delta$ scales as $\sqrt{T}$, the spin only responds to the average field, recovering the static approximation:

$$
R \approx \exp \left|\int_{0}^{\beta} \frac{1}{2} \Delta(\tau) d \tau\right|, \quad|\Phi| \ll \pi
$$

Let us now consider a more general extended Hubbard Hamiltonian with $N$ orbitals per site:

$$
\begin{aligned}
H= & H_{\text {band }}-\frac{1}{N} \sum_{i} \sum_{j \neq i} J_{i j} \mathbf{S}_{i} \cdot \mathbf{S}_{j}-\frac{1}{N} \sum_{i} J_{i i} \mathbf{S}_{i} \cdot \mathbf{S}_{i} \\
& +\frac{1}{4 N} \sum_{i} U n_{i}^{2}
\end{aligned}
$$

where

$$
H_{\mathrm{band}}=-\sum_{i, j} \sum_{a=1}^{N} \sum_{s=\uparrow}^{\downarrow} t_{i j} c_{i a s}^{\dagger} c_{j a s}
$$

and $\mathbf{S}_{i}$ and $n_{i}$ are total charge and spin operators on site $i$,

$$
\mathbf{S}_{i}=\sum_{a=1}^{N} \mathbf{S}_{i a}, \quad n_{i}=\sum_{a=1}^{N} n_{i a} .
$$




$$
\begin{aligned}
& \text { Applying the Hubbard-Stratonovich transformation with } \\
& \text { gives }^{17} \\
& Z \propto Z_{\text {band }} \int \prod_{i} \mathcal{D}^{3} \Delta_{i}(\tau) \mathcal{D} w_{i}(\tau) \\
& \times \exp \left\{-N \beta\left(V_{0}[\Delta, w]-k T \ln z[\Delta, w]\right)\right\}, \\
& \text { and } \\
& \begin{aligned}
V_{0}[\Delta, w]=\int_{0}^{\beta}[ & \sum_{i, j} \frac{1}{4} J_{i j}^{-1} \Delta_{i}(\tau) \cdot \Delta_{j}(\tau) \\
& \left.+\sum_{i} \frac{1}{4} U^{-1} w_{i}(\tau)^{2}\right] d \tau
\end{aligned} \\
& z[\Delta, w]=\left\langle\mathcal{T} \exp \int_{0}^{\beta} \sum_{i}\left[\boldsymbol{\Delta}_{i}(\tau) \cdot \mathbf{S}_{i 1}(\tau)+i w_{i}(\tau) n_{i 1}(\tau) / 2\right] d \tau\right\rangle_{\text {band }}
\end{aligned}
$$

The operators $\mathbf{S}_{i 1}$ and $n_{i 1}$ refer to a single band. $A(\tau)$ denotes explicit time dependence of the operator $A$ with respect to $H_{\text {band }}$. The degeneracy $N$ appears only as a factor in the exponent in Eq. (29). If the $\mathbf{S}_{i a}$ are spin operators, and $t_{i j}=U=0$, the Hamiltonian (26) becomes a Heisenberg model. If $J_{i i} \gg \sum_{j \neq i}\left|J_{i j}\right|$ the spins on each site couple strongly, and low-lying excitations are those of the spin- $N / 2$ Heisenberg model. The preceding discussion remains valid, with the phase of each path multiplied by $N$. It is possible to integrate out the finitefrequency components of the exchange field, leaving an exact static potential. This was the basis of an attempt at a $1 / N$ expansion: the leading term in the expansion of that potential is static approximation. ${ }^{17}$ However, the negative-weight paths are important: the time-averaged field $\int_{0}^{\beta} \Delta(\tau) d \tau$ has no positive-definite distribution at low temperature, just as two noncollinear spin components have no positive-definite Wigner distribution.

The situation for the Hubbard model (obtained when $J_{i j}=0$ for $i \neq j$ ) is less clear cut. The state space of the Heisenberg model is a product of spheres $\left\{\mathbf{n}_{i} ;\left|\mathbf{n}_{i}\right|=1\right\}$. The state space of the Hubbard model is of higher dimensionality. However, for narrow bands the important low-energy excitations are often spin fluctuations and the geometrical approach may still provide insights. Consider the ground sate of the noninteracting electron system in a static field $\left\{\Delta_{i}\right\}$, neglecting the charge field $\left\{w_{i}\right\}$ for simplicity. In the spirit of spin density functional theory, one can label the ground state by the local moment on each site, or equivalently a point in the manifold $\left\{\left\langle\mathbf{S}_{i}\right\rangle ;\left|\left\langle\mathbf{S}_{i}\right\rangle\right|<\frac{1}{2}\right\}$. A slowly varying field $\left\{\Delta_{i}(\tau)\right\}$ transports the point around the manifold as in the Heisenberg model. The manifold does not, however, exhaust Hilbert space. Discontinuities in $\Delta_{i}(\tau)$, not excluded from the functional integral (29), produce electronic excitations. Neglect of these excitations would lead to a solution of the Hamiltonian in a restricted basis set. It remains to be seen whether such a scheme is a productive extension of the static approximation.

This paper has exhibited features of the sign (or phase) problem. It is not the intention to solve the problem but rather to gain insight into its cause: at low temperatures, the electron or spin system can follow the field around a large loop, developing a phase factor. At high temperatures the spin only responds to the average field, recovering the static approximation. This may lead to a means of classifying or combining paths in such a way as to circumvent the sign problem. The geometrical approach also points towards an improvement to the static approximation that will reintroduce the most important quantum fluctuations.
${ }^{1}$ W. E. Evenson, J. R. Schrieffer, and S. Q. Wang, J. Appl. Phys. 41, 1199 (1970).

2 J. H. Samson, in Proceedings of the International Conference on the Physics of Transition Metals, Darmstadt, July 1992 [Int. J. Mod. Phys. B (to be published)].

${ }^{3}$ M. V. Berry, Proc. R. Soc. London, Ser. A 392, 45 (1984).

${ }^{4} J$. Moody, A. Shapere, and F. Wilczek, Phys. Rev. Lett. 56, 893 (1986).

${ }^{5}$ O. K. Vorov, in Topological Phases in Quantum Theory, edited by B. Markovski and S. I. Vinitsky (World Scientific, Singapore, 1989), p. 112.

${ }^{6}$ A. Angelucci and G. Jug, Int. J. Mod. Phys. B 3, 1069 (1989).

${ }^{7}$ J. W. Negele and H. Orland, Quantum Many Particle Systems (Addison-Wesley, Redwood City, CA, 1988).

${ }^{8}$ K. S. Chana, J. H. Samson, M.-U. Luchini, and V. Heine, J. Phys. Condens. Matter 3, 6455 (1991).
${ }^{9}$ E. H. Loh, Jr., J. E. Gubernatis, R. L. Scalettar, S. R. White, D. J. Scalapino, and R. L Sugar, Phys. Rev. B 41, 9301 (1990). ${ }^{10}$ J. Hirsch, Phys. Rev. B 31, 4403 (1985).

${ }^{11}$ S. Fahy and D. R. Hamann, Phys. Rev. B 43, 765 (1991).

${ }^{12}$ A. Muramatsu, G. Zumbach, and X. Zotos, Int. J. Mod. Phys. C 3, 185 (1992).

${ }^{13}$ C. A. Macêdo and M. D. Coutinho-Filho, Phys. Rev. B 43, 13515 (1991).

${ }^{14}$ S. Leibler and H. Orland, Ann. Phys. (N.Y.) 132, 277 (1981).

${ }^{15} \mathrm{E}$. Fradkin, Field Theories of Condensed Matter Systems (Addison-Wesley, Redwood City, CA, 1991), Chap. 5; E. Fradkin and M. Stone, Phys. Rev. B 38, 7215 (1988).

${ }^{16}$ Y. Aharonov and J. Anandan, Phys. Rev. Lett. 58, 1593 (1987).

17J. H. Samson, Phys. Rev. B 30, 1437 (1984); J. Magn. Magn. Mater. 54-57, 983 (1986). 